\title{
Investigating teachers' work with multiple knowledge resources in local curriculum development
}

\section{Eli Tronsmo}

Department of Education, University of Oslo, Postboks 1092 Blindern, 0317 Oslo, Norway

Email:eli.tronsmo@iped.uio.no.

Author bio

Eli Tronsmo is a Ph.D. candidate in the Department of Education, University of Oslo. She writes on teachers' knowledge work, and has a special interest in the ways in which professional groups handle new knowledge challenges related to changing conditions for their work and learning.

Acknowledgements

I wish to thank the participating teachers for the research opportunities enabled by their work. I am also grateful to Professor Monika Nerland, Professor Andreas Lund, Hege Hermansen, and participants in the ExCID research group for helpful comments to earlier versions of this paper. 


\title{
Investigating teachers' work with multiple knowledge resources in local curriculum development
}

\begin{abstract}
This paper investigates how teachers handle dilemmas generated by the multiplicity of knowledge resources in contexts of local curriculum development. In a study using an ethnographic approach, one team of lower secondary school teachers in Norway, mandated to develop a school based curriculum, was followed closely over a year. Drawing on socio-material perspectives, data from six team meetings were analysed to explore how the team reached decisions in their efforts to develop a subject curriculum. While previous research has paid less attention to what the presence of a diversity of knowledge resources 'does' with the epistemic dimension of teachers' curriculum work, the present study shows that teachers' engagement with multiple knowledge resources created dilemmas but also greater scope for action in decision-making situations. These processes, however, bring new responsibilities to the fore which require agency from the teachers and should be acknowledged in current discussions of teachers' work.
\end{abstract}

Keywords: local curriculum development; teacher collaboration; epistemic responsibilities; knowledge resources; sociomaterial analysis

\section{Introduction}

The settings in which teachers carry out their work have increasingly become more materially dense. Teachers engage with not only people but also a multitude of tools, procedures, texts, models, research evidence, and technologies. While some of these resources originate from research, others are derived from local practice, policy, and global flows, such as curriculum models and technological monitoring systems, which are shared and circulated across organisational and national borders. Hence, instruments, manuals, standards, and methodologies entering the teaching profession act as linkages between the fields of work and knowledge production (Nerland and Jensen, 2014). Material devices are, however, not neutral. Rather, they incorporate knowledge and beliefs taken from where they originate, and function as what Scott (2014) describes as 'carriers' of particular logics and intentions (e.g. concerning examples of how to act) (März, Kelchtermans, and Vermeir 2017) and are, thus, potential knowledge 
resources that are mobilised when applied in local contexts. Although knowledge resources embody specific ways of understanding and putting knowledge to use, they do not determine practice but pave the way for interpretive flexibility (Cho and Wayman, 2014). For teachers to navigate this landscape of resources, they require the ability to negotiate different concerns and to handle the simultaneous presence of varied logics, knowledge, and representations (Fransson and Grannäs 2013; Hirsh 2014; Jonasson, Mäkitalo, and Nielsen 2015; Singh Märtsin and Glasswell 2014). This, in turn, challenges our understanding of the teaching profession and what it encompasses in a high-tech, international, knowledge-based society. However, we have no solid understanding of how tensions between logics of knowledge embodied in materiality emerge nor of the kinds of dilemmas they may generate.

One of the instances in which dilemmas and tensions may come to the fore in teachers' knowledge work is in local curriculum development, as such work occurs at the intersection of multiple social and epistemic concerns (e.g. Ben-Peretz and Flores 2018; Hargreaves 2003) and involves complex problem-solving and decision-making (Boschman, McKenney, and Voogt 2014). Increased attention has been given to teachers as curriculum designers and to how this work both presents challenges and provides learning opportunities for teachers (Voogt et al. 2016). However, we lack a solid understanding of the role of knowledge resources, the dilemmas and negotiations that these may trigger when decision-making is an explicit goal, and the strategies that teachers employ to handle such dilemmas.

To get to the heart of this issue, this paper examines the dilemmas that follow in the wake of the multiple available knowledge resources used during teachers' decisionmaking processes in the context of school-based curriculum development.

This study follows a team of lower secondary school teachers in Norway as they attempt to develop a subject curriculum for their school. The case is suitable for this purpose because it allows for the examination of the dilemmas that may emerge and because this group of teachers was mandated to develop a school-based curriculum.

Norway provides an interesting context in which to examine such processes, as teachers are given responsibility for local curriculum development. School-based curriculum construction is framed by national standards and guidelines that are defined by the Ministry of Education, leaving extensive responsibility for teaching professionals 
to determine how to develop and organise a common curriculum at the local level (Czerniawski 2011; Hatch 2013).

This examination employs analytical resources from a body of socio-material approaches (Fenwick, Edwards, and Sawchuk 2011; Gherardi and Strati 2012; Nespor 2003) which emphasise the materiality of knowledge and the ways in which its enactment generates effects in practice. As well as attending to these material features, teachers' agency in the ways of mobilizing these resources is recognized and paid analytical attention to. Before presenting the theoretical framework, I first provide a brief review of the research on dilemmas in teachers' curriculum work.

\section{Curriculum work as imbued with dilemmas}

Research has shown that many forms of dilemmas exist in teachers' curriculum work. These dilemmas are related to tensions arising in the wake of high-stakes testing and assessment issues (Singh et al. 2015), contradictory pedagogical concerns (Jonasson et al. 2015; Wall 2017), and the introduction of new technologies and standards (Hardy and Lewis 2018).

One strand of research has investigated the ways in which the complexity of new educational demands is enacted by teachers by drawing on perspectives from institutional theory. This work has investigated, for instance, how teachers negotiate policy dilemmas (e.g. Hirsh 2014; März et al. 2017; Priestley, Minty, and Eager 2013; Wall 2017) in the context of curriculum work. According to these studies, teacher dilemmas often arise at the intersection of politics, bureaucracy, and profession. However, these studies pay limited attention to the epistemic dilemmas that arise.

Another strand of research has investigated how global education networks have enabled the circulation of instruments, ideas, and technologies by drawing on critical policy studies (e.g. Ceulemans, Simons, and Struyf 2012; Fenwick, Mangez, and Ozga 2014). This work suggests that knowledge-based technologies introduce a variety of sometimes contradictory expectations into teachers' curriculum work in ways that create dilemmas. For instance, digital data use in education may be tied to external accountability demands, which, in the words of Hardy and Lewis (2017), position students 'less as students and more as data' while simultaneously incorporating intentions of differentiated instructions to provide individualised student support. Such studies also show that practices change when new ways of ordering information arise, 
be it through assessment programmes (Remesal, 2011), learning analytics (Hardy and Lewis, 2017), or attempts to standardise, which trigger specific practices and tensions. We need a better understanding of how competing logics triggered by knowledge resources may lead to epistemic tensions and how these tensions are handled by teachers.

A third strand of research has investigated how teachers' work with purposefully designed objects requires extensive processes of collective sense-making (e.g. Coburn 2001; Hermansen and Nerland 2014; März et al. 2017). These studies unravel the processes of interpretative negotiation between the meaning systems of the materiality and its users (Vermeir, Kelchtermans, and März 2017), as well as the recontextualisation that this implies (Singh et al. 2015). This work has shown how sensemaking processes also involve the adaptation and redesign of materials, as in studies of teachers' curriculum design, such as written plans, assessment rubrics, and activity templates (Boschman et al. 2014). As shown in a review of research on teachers' capacity for data-driven decision-making (Datnow and Hubbard 2016), the scope of new initiatives and accompanying knowledge resources places increasing demands on teachers to master them. The authors conclude that such processes are not straightforward and require an in-depth understanding of subject matter, curriculum, and how students learn in order for teachers to make use of resources, such as, in their case, student data.

What all these studies seem to illustrate is that materialised representations of politics and knowledge are not neutral and that they play an important role in teachers' judgments and decision-making processes. While previous research devoted attention to dilemmas emerging from ideological tensions (e.g. Apple 2007; Ball 2005), there is a need to gain a better understanding of how instruments, standards, and templates for curriculum work incorporate knowledge and notions of how they should be applied. The incredible volume of new knowledge resources used to improve education creates a historically new situation in which teachers' work is potentially more complex and contested while also imposing greater responsibility on teachers to deal with the dilemmas generated by them.

This paper examines epistemic dilemmas that arise in teachers' curriculum work and the strategies employed to handle them. The following research questions guide the analysis: 
1) What kinds of epistemic dilemmas emerge in teachers' local curriculum work with multiple knowledge resources?

2) How are such dilemmas handled as socio-material processes in teachers' decision-making?

To explore these questions, we need a perspective that accounts for the constitutive role of knowledge resources in social practices. This implies that it is not sufficient to focus on individuals alone.

\section{Analytical perspective}

What relationships do we need to understand in order to pursue how dilemmas arise, are handled and enable decision-making? In order to open up the material aspects of activity, this paper draws on resources from socio-material perspectives. In this regard, materiality could, for instance, be textbooks and virtual spaces, protocols and timetables, syllabuses and standards, assessment criteria and test scores, white boards and staff rooms, or algorithms and policy documents. Hence, a socio-material perspective focuses attention on the vast number of resources that embody the knowledge of a given domain or across domains.

Second, socio-material theories pay attention to the relationships between social and material components, as well as the manner in which both subjects and objects continually entangle (Fenwick et al. 2011; Vanden, Buverie and Simons 2017) through material and discursive components. Instead of studying objects and humans separately, attention is directed towards how they work together. In this study, the focus is on matters such as technologies, subject-specific and pedagogical discourses, and various educational devices. Thus, the focus is on the relationship between things as emergent phenomena and how these things influence and alter one another in ways that are continually opening and foreclosing new spaces and possibilities. Such a relational approach takes into account how spaces emerge when objects relate (Massey 2005). Here, this refers to how spaces for action emerge when various resources are assembled and acted upon.

Third, socio-material theories tend to be used to understand capacities for action and the relationships between subjects and objects, including knowing-in-practice (Gherardi and Strati 2012), as performed into existence. Applied to this study, the 
meanings of assessment, subject content, competence goals, and so forth are neither timeless nor space-less attributes of these curriculum elements; rather, in Nespor's terms, they are 'processes of transformation, compromise or negotiation' $(2003,96)$. Each element has stories, values, and logics of knowledge embedded within it. From this, we can understand teachers' work with knowledge resources as the handling of materiality that carries 'inscriptions' (Markuskaite and Goodyear 2017) and importantly, as a handling which requires active sense-making. This means, for instance, that assessment criteria or a specific teaching method undergo change every time they are acted upon and applied in new local contexts through processes that Asdal (2015) has called 'modifying work.'

To shed light on the ways in which materials are integral in shaping the particular decisions that are enacted in local instantiations of practice, there is a need for a more detailed account of how to understand the various knowledge resources that teachers tap into. While some knowledge resources aspire to have global or 'generic' validity, others claim to be more contexts dependent.

One way of understanding the varied logics inscribed in materiality is by turning to knowledge production itself, which varies with respect to different 'epistemic orientations' (Kastenhofer 2007). While some resources may originate from what Kastenhofer conceptualises as a control-oriented epistemic environment-meaning that it is marked by highly standardised and controlled scientific logics —others originate from one that is complexity oriented, meaning that it aims to capture the complexity of living systems. Still other resources may originate from experience-oriented knowledge-producing environments, which are marked by closeness to the context and the 'problem at hand.' From this, we can conceptualise international comparative student tests as coming from a control-oriented logic, while studies of students' home environments incorporate a complexity-oriented logic. Hence, following Kastenhofer's heuristic and drawing on the concept of 'epistemic orientations,' this study pays analytical attention to the epistemic logics inscribed in materiality that 'travel' from the field of knowledge production to the field of application. The aim is to understand how knowledge of various domains is materially manifested in resources, as well as the ways in which these become connected in new ways. I look for the spaces of action that occur when resources carrying different epistemic orientations are combined, assembled, and made sense of for decision-making purposes in teachers' curriculum construction. Space 
is understood here as a relational category (Fransson and Grannäs 2013) that constitutes rationalities for actions with orientations. It should be emphasised, however, that there is no direct transfer between the epistemic orientations incorporated into knowledge resources and the realisation of their inherent logics in professional practice.

When multiple epistemic orientations incorporated into manuals, procedures, and tools enter teachers' work, this may potentially generate dilemmas (Fransson and Grannäs 2013) — for instance, if resources that aspire to have generic validity for all students' learning meet educational resources that target specific local groups. Hence, to understand the complexity of decision-making processes in teachers' curriculum work, we need to account for the different and potentially competing forces of knowledge resources. From this perspective, I analyse how knowledge resources guide and shape spaces for action and how teachers approach and ascribe meaning to such resources in decision-making processes. Figure 1 depicts the interplay between knowledge resources with orientations and the 'doings' of teachers and how this opens up spaces of action and provides grounds for decision-making.

Figure 1 Decision-making as process. The figure shows interplay between school context and teacher level. The curved arrows depict the dynamics between teachers'sense making and knowledge resources activated.

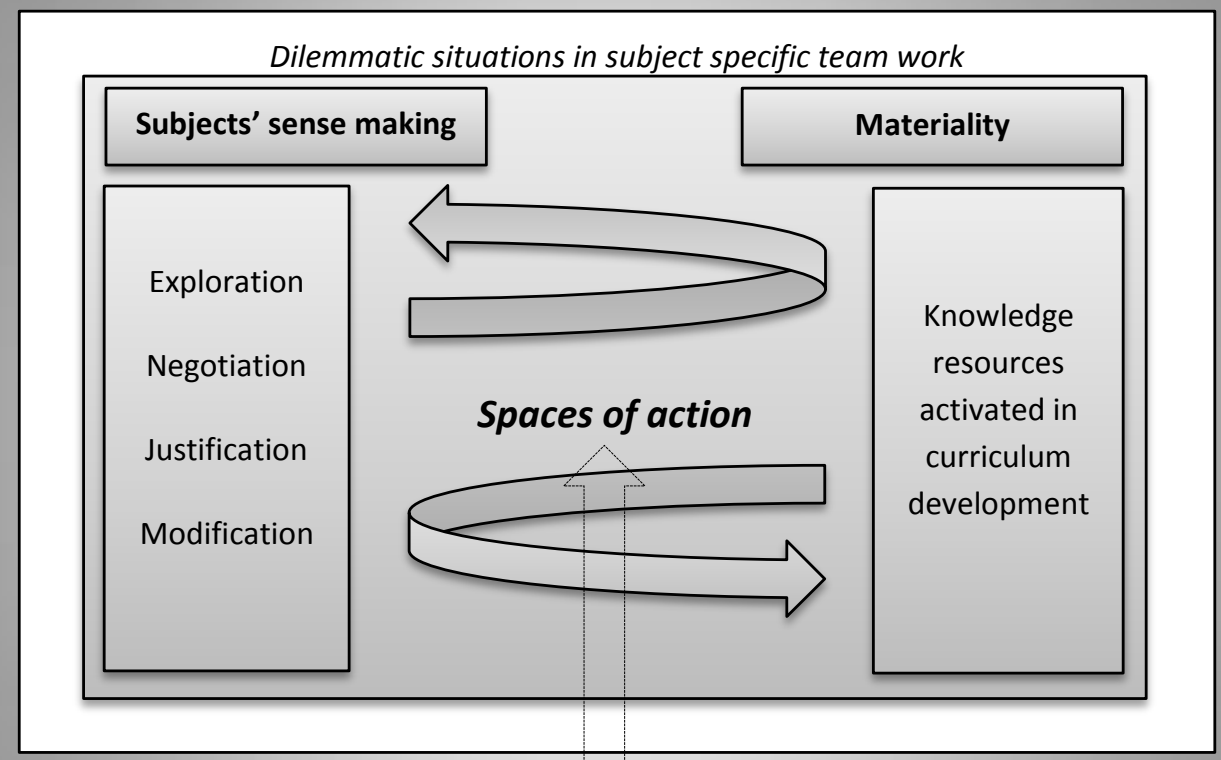

Knowledge resources circulating in the school context

Decision-making 


\section{Design and methodology}

This study adopts a qualitative methodology approach that is centred on the observations of collaborative curriculum construction in a subject-specific team of social science teachers at one lower secondary school in Norway.

\section{Empirical setting and data}

The school was selected based on purposive sampling, which is a strategy that aims to 'identify and select the information-rich cases' (Patton 2002). At this school, local curriculum work had been prioritised at the organisational level based on a request from the staff to broaden their general curriculum understanding. Consequently, all the teachers at the school had been organised into subject-specific groups mandated to develop a subject curriculum for their school with a validity framework of three years. Most teachers participated in two subject groups of approximately six teachers each from different grade levels (8-10). The current analysis is based on the meetings and work of the social sciences subject group. A group leader, appointed by the principal, chaired the meetings and engaged with school management weekly. The meetings had a structured agenda with the items introduced in advance by the group leader. A meeting report was distributed to the group after each meeting.

In line with the capacity of ethnographic approaches to allow for data generation across organisational sites and levels (Yanow, Ybema, and Hulst 2012), data were collected to observe epistemic dilemmas at both the school level and the level of the teachers' joint practice. The data corpus was based on observation, interviews, documents (e.g. the school's strategy documents, distributed supplies, and meeting reports), and photos. The observations foreground the voices of the participants, albeit interwoven with the tracings of the material activities. I traced the creation and development of the curriculum plan via the various versions and associated resources and templates stored in a shared online space. Observations and interviews were recorded with a video camera and supplemented with handwritten field notes that were typed up after each visit and stored in a Fieldwork Activity Log. Video recordings were used to enable consideration of visual and material aspects, as well as speech, while still providing a record of the in situ action (Derry et al. 2010). The meaning of in situ 
actions was not always self-evident; therefore, further data from interviews or group discussions were collected to unpack the way in which the participants understood tensions and justified their decisions. Additional data were gleaned from other meetings (e.g. grade-level meetings and planning days) and school documents. Video observations and interviews were transcribed verbatim. To trace the complexity of the subject curriculum, data collection occurred over the course of one school year (20152016). Six meetings of the subject-specific group that was engaged in the work of collaboratively developing a social sciences curriculum for their school were analysed in depth.

\section{Analytical strategy}

In line with the theoretical perspectives outlined above, the aim of the analysis was to gain an understanding of the following issues: first, the kind of materiality assembled in situations of collaborative curriculum construction; second, the dilemmas emerging in relation to the materiality enacted by the teachers; and third, the knowledge processes underlying the teachers' decisions.

The analysis was conducted in three steps. First, through repeated readings of the transcripts and field notes and the viewing of the video materials and photos, the ethnographic data were approached using content mapping (e.g. framing of problems, knowledge resources that mobilised emerging tensions, and modes of justification, such as when specific authorities were referred to) that was formulated based on my theoretical and methodological interests. These categories were refined while readings of the material were inductively generated around emerging themes (Ryan and Bernard 2003). Second, following Andriopoulos and Gotsi (2017), I looked for emerging tensions and opposing elements across the data — that is, at both the organisational and interactional levels. The social and material interactions that occurred within the teacher team were analysed, emphasising the role of materiality. In line with Read and Swarts (2015), I looked for the origin of knowledge resources or what knowledge forms the resources had inscribed. I looked for language indicators (Andriopoulos and Gotsi 2017) such as 'on the other hand,' 'what's really important is ...,' 'is this the right way to ..., ', and justifications that may be indicative of epistemic tensions in the transcripts (e.g. tensions between different guidelines on how to formulate learning objectives or about assessment issues). Third, once I determined that these episodes warranted further 
analysis, I used the concepts of negotiation, justification, and modification as sensitising means (Czarniwska-Joerges 2007) to unpack the details of these episodes and conduct an in-depth analysis. In several rounds of the analysis, raw data and interpretations were presented to co-researchers in joint data sessions, and three researchers systematically compared their interpretations.

Some recurrent patterns emerged. These are presented with reference to various knowledge resources entering at the school level, which triggered dilemmatic situations and two empirical episodes of social interactions in situations of curriculum construction in which tensions were present.

\section{Findings}

This section turns to the findings. The first part addresses findings from the school level with regard to the richness of the material knowledge resources present. The second part reports on findings from the interactions in the teacher team, focusing first on the materiality activated and what it initiated and, second, on what the teachers 'did' with this materiality (negotiation, justification, and modification) to enable them to make decisions.

\section{Multiple knowledge resources and potential tensions in the school context}

Although the curriculum work was framed by national guidelines for an outcome-based plan with assessment criteria in conjunction with its various content items, there was extensive space within which the teachers could define the aims, contents, and methods of teaching and learning to be incorporated into the subject curriculum. Within this space, a range of different artefacts, technologies, and procedures were at play, many of which had different purposes and logics. These included, for example, assessment forms, methods of reading instruction, or the purposes of a specific subject. All of these potentially came to inform the teachers' decision-making to various degrees, but they also caused dilemmas. Moreover, among these resources, some originated from governmental agencies, some from research agencies, and some through partnership agreements with technology companies and other private businesses. Still others were derived from local authorities or the school itself. Hence, the number of resources at the school level was comprehensive, multifaceted, and rooted in different systems of relations and knowing. For example, the 
use of digital management systems that systematised and analysed information about students' results and learning processes was extensive. Such systems play an increasingly important role in education, where they are typically used for the purposes of informing decision-making. The headmaster explained as follows:

We have a large municipal database that provides us access to patterns in student results throughout primary and lower secondary school. Various parameters can be added. But now, we are considering a new system that gives access to even more varied data-for example, parents' educational background and other parameters that we may want to compensate for through our teaching.

These digital systems generate statistical and criterion-based reports from large amounts of data that are derived from various sources (including student surveys, school-based mapping tests, and national and transnational tests) to set local goals and measures for assessing and recording student progress. As a knowledge resource for teachers and managers, digital analytics functioned as a navigational tool that mediated the school's understanding of students in certain ways, transforming them into aggregates and numbers that could be moved around and combined with other numbers elsewhere as statistical expressions of comparable groups. These technologies generated framings of students as de-centred subjects, and from this, the established categories formed the basis of the interventions and decisions through the control of variables.

Other resources that were circulated at the school included a curriculum development template. This was developed through collaboration between teachers and school managers a year before the observation started, and it covered the various curriculum components, such as teaching methods, subject content and skills, assessments, and excursions. The various categories in the template were linked to other objects related to the subject content, instructional ideas and concepts of learning originating from the research communities, the Directorate of Education, external consultants, professional networks, and other knowledge-producing environments and practices. A central category in this template was a sociocultural component to improve the forming aspects of the subjects, and it concerned methods for ensuring 'whole student' development across multiple domains.

Furthermore, a number of available guidelines and conceptual resources on how to approach a broad range of assessment practices had been generated from a 
comprehensive national effort in the field of assessment. Concepts such as 'Assessment for Learning' (AfL), 'backwards design,' 'teaching with big ideas,' and 'flipped classrooms' were disseminated internationally in close connection with a myriad of material templates, tests, individual learning plans, and so on being fed into the school.

Another 'whole student' resource at play was a school-based agreement as to how to approach students. This 'student view,' as they called it, comprised strategies for addressing students' social and emotional development and acted as a resource for teachers that took into account students' various challenges and stories, as elaborated by the headmaster:

The teachers' mandate is far greater than preparing, assessing, and conducting teaching. We have all kinds of people integrated in our school ... people with different backgrounds and mental health challenges. This places a great demand on the staff.

Hence, the students in this school were not only constructed as predefined categories, as in the digital management systems, but were also generated as 'whole people' falling into complex and unpredictable categories. These two different framings of 'the student' worked from different premises but coexisted in the school's strategy documents, as did different premises for understanding notions of 'assessment' and/or 'learning.'

As these examples illustrate, the teachers' curriculum development took place in a school context that was marked by multiple socio-material relations. These included student data, assessment tools, educational research, school priorities, policies, and various regulations, which together generated a contemporaneous plurality comprising different epistemic orientations and expectations. These were all products of various knowledge-generating practices, some of which were developed to have global validity, such as models of taxonomies from the learning sciences, while others were developed to have more limited and contextual applications. Although contradictions between different ideas of validity inscribed in resources coexisted at the school, these contradictions were not 'offloaded' at the organisational level but were left for the teachers to handle. What happened, therefore, when the teachers made decisions within these frameworks? In the following section, I examine spaces of actions to investigate how the team of social science teachers handled assemblages of knowledge resources, 
the dilemmas and tensions these generated due to being rooted in different epistemic orientations, and the ways in which such dilemmas were resolved and materialised into a shared subject curriculum.

\title{
Exploring processes of decision-making
}

Throughout the six meetings analysed, various suggestions and solutions were discussed within areas such as teaching methods, the selection of subject content, the formulation of learning goals, and assessment issues. A meeting report summarised discussions and decisions after each meeting and gave the teachers a meta-view of their own curriculum development. As Rob, one of the teachers, explained in an interview,

\begin{abstract}
We must recognise that in processes like these, we cannot be one hundred percent satisfied. You must take a little and give a little. Take the curriculum template: it generated a lot of discussions about what to put here and there, columns and competence goals, and so on (...). It was only in the last two or three meetings that there was a 'Yes, we will be able to use this!' But it has been difficult, because the terrain has been unclear, and it takes time to understand the components together.
\end{abstract}

Work was also conducted between meetings, and participants used a shared online space (available to all staff) to add resources and new ideas for discussion in forthcoming meetings.

Although both episodes presented below illustrate tensions arising, the starting point for the dilemmatic situation in the two data excerpts was different. In the first episode, the tension came from the activation of knowledge resources 'from outside,' which were intended to have global validity but triggered negotiation. In the second episode, the school's idea of interdisciplinarity came into play. These episodes were selected because they show forms of knowledge resources at play and the emergence of dilemmas. They are from the fourth meeting and provide examples of how discussions alternated between dilemmas and solutions that were close in time and are, therefore, useful for addressing the research questions.

\section{Negotiating taxonomies. Episode 1.}

Immediately after the last student has left the school building, six social science teachers come together in an empty classroom and push six desks together to form a 
provisional meeting table. As they arrive, the teachers are logging onto their laptops and pouring themselves coffee before sitting down. Today, one of the themes on the agenda is assessment issues and the question of whether learning progression follows generic patterns that should be taken into account in the curriculum. The national AfL programme has provided them with extensive resources and knowledge of the different taxonomies which are mentioned in the school's strategy document as being among the many useful resources. These are, however, only sketches-not very fine-grained suggestions - and require further adaptation.

The following excerpt takes place forty minutes into the meeting, when they attempt to come to grips with a representation of assessment criteria across subjects. Attention is directed towards a representation of the current version of the subject curriculum projected on the shared screen. They alternate between the columns for Teaching (subject content), Skills, and Assessment, and the discussion centres around the question of how to align the Skills and Subject content while simultaneously attempting to systematise descriptions of progression in student learning. The expected progression in student learning, however, can be expressed in a curriculum in several ways. Now, they focus on 'action verbs' in use when deciding on formulations of learning objectives. For example, they investigate whether the ability to 'compare' is a form of 'discussing.' In the process of elaboration, Stephen, a tenth grade teacher, gets up and starts to draw a triangle on the blackboard next to the screen, and he says the following:

Stephen: Maybe we could draw a triangle that we divide into three, just like the classical model, where we place the arguing parts on top and the recalling parts at the bottom?

Rob: But, that's Bloom ... !

Stephen: Yes, exactly. That's Bloom's taxonomy ... just like that. The point is that we categorise the verbs we select based on that model. I don't know whether it's a good idea or what you think.

Oliver: I have my views about it, but the others can give their input first.

James: Well, it's possible to recall at different skill levels, too. Maybe we could make a different model ... Because it's possible to recall at a high skill level, and you can recall at a low level. You can discuss at a high skill level, and you can discuss at a low level. (...) 
Stephen: Yes, exactly. That's why we categorise them [the verbs].

Oliver: But now we have two different ways to categorise. What you do there is one way [referring to Bloom's taxonomy], and combining the different verbs is a different way_-for example, by asking 'What characterises good arguing in the eighth, ninth, and tenth grades?'

I am not necessarily opposed to your suggestion, except that I think this decision is terribly difficult. We must try to see what it implies. And, so far, we have tried in some other way.

After extensive discussion and trying out different taxonomies over two meetings—first, by clustering verbs in a different manner from Bloom 's taxonomy suggests - they finally come to a decision according to a standard by means of which all tasks are specified by characteristics of 'high achievement.'

What knowledge processes formed the basis for this decision? To answer this question, I will first turn to the knowledge resources at play. In this excerpt, several material and conceptual resources come to the fore. In addition to Bloom's taxonomy, a locally developed standard to classify degrees of student achievement is brought to the table (Oliver's final utterance). Concepts such as 'skill level' and 'goal achievement' are invoked, as is the implicit stance of classifying verbs to express competence goals, as well as the very idea that skill levels are observable. This socio-material environment generates tensions between different ways of framing the problem at hand.

First, with Bloom, a set of classification systems is raised. Bloom's taxonomy originates in the learning sciences and has been discussed, contested, and further developed before arriving at the teachers' table in a modified version. In line with other knowledge resources, it is inscribed by theoretical knowledge, expectations, and controversies. The value of Bloom's taxonomy rests in part on its ability to reduce complexity by enabling assessment of the degree of goal achievement based on a fixed standard that can be verified, compared, and shared across subjects and teaching situations. However, a different standard, which was newly developed in the school and which provides another way to classify degrees of achievement, is also activated. This offers an alternative way of thinking about student progression based on logic which underscores the importance of context and other qualities in standardising the 
descriptions of expected learning outcomes. Tension appears between different conceptions of learning. But how is this tension handled by the teachers?

The presence of Bloom's taxonomy brings about negotiations among alternatives connected to broader discourses in the old learning sciences. For example, James argues that learning does not necessarily proceed linearly towards more abstract levels, as Bloom's taxonomy implies, but (the development of) skills might as well be evoked in practical contexts. This leads to a need for justifications and exploration. 'To recall' may be expressed at different levels, which implies that the placement of verbs in different cognitive categories is not necessarily the best criterion for building a standard, because what, in some cases, is described as low goal achievement—for example, 'to exemplify' - may mean the opposite in a different context.

Finally, statements such as 'Maybe we can make a different model' and 'We must try to see what it implies' illustrate that the teachers invoke their own experiences in addition to the resources generated from the 'outside.' At this stage, the inclusion of an alternative to Bloom's taxonomy, the school-based model, opens up a space for negotiation in which different models of classifying degrees of student achievement are evoked. This space enables the teachers to explore various options and to transform the emerging tension into an alternative route. Before they can come to a final decision, they must explore the implication of following one standard over the other. Thus, the tension creates an explorative space for seeking out alternative possibilities. However, this not only implies that the teachers seek out the 'best' among the alternatives but also requires them to unveil the underlying premises and logics that inhabit the various standards that are evoked. When Bloom enters with a certain control orientation, the teachers' strategy is to activate experience-based resources as a 'counter force.' This illustrates modifying work and agency in the sense that the teachers actively find ways to transform the issue at hand and to turn it into a decision.

To summarise, the indeterminacy that occurs is not a static configuration of opposing elements, but the involvement of multiple concerns, discourses, and actors. Bloom's taxonomy aspires to have a global value but collides with a request to maintain a broader approach to framing the criteria for assessing students' learning progression. However, at the intersection in which a material carrier of one tradition faces the enactment of a quest for a new model, the teachers' construction of the local curriculum progresses, in part, as it extends beyond current knowledge and, in part, as it challenges 
and transforms standards. What enables the decision are the spaces that emerge when different avenues for action are negotiated and modified.

I now turn to the next episode, which illustrates a dilemma that the teachers encounter while trying to arrive at a conclusion regarding questions about interdisciplinary collaboration.

Justifying ideas of interdisciplinarity. Episode 2.

The discussion on taxonomy has come to a preliminary conclusion, and they are now discussing 'interdisciplinarity,' which is the final theme on today's meeting agenda. Interdisciplinarity is mentioned in the school's strategy paper for curriculum development: 'local curriculum construction should aim to focus on interdisciplinary topics that can be thematically coordinated across subjects.'

The teachers' attention is directed towards the column for competence aims in the plan on the whiteboard. They ask whether some of the competence aims they have formulated so far also apply to other subjects. They consider whether they might be able to develop an interdisciplinary project on terrorism in collaboration with the English Department or maybe with religion. Soon, the interaction moves to assessment issues. If they are to merge instruction and competence aims across subjects, should assessments be merged as well? The number of assessment situations in each subject during a school year is extensive, and several teachers have argued that the number is a burden for the students and should be reduced.

Stephen: The sooner we get started with interdisciplinarity, the faster we can reduce the number of assessment situations for the students. We've got this in place already, so, actually, from next year perhaps, we can get away with two, maybe three, grades per assignment, submission, or test. As for the sake of the students, we should give priority to interdisciplinarity.

Oliver: I agree. I just think it's the hardest part. But I guess it's easier when other things are in place. That's what I mean.

Stephen: Well, someone should start ...

Oliver: But we've been prohibited from carrying out [assessment] interdisciplinarity between languages and other subjects, unless the students themselves want to. Just so everyone is aware of it. I know it. It was taken up in plenary. We cannot run English and social sciences in combination and then give a social 
sciences grade on it.

Rob: When was this taken up in plenary?

Oliver: We discussed a specific case, because some of us had designed a test combining second language and religion. And in this regard, we were in contact with the EPCS ${ }^{1}$ to discuss the case, and they said that it's not feasible. Their point is that if you have a student, not necessarily with a literacy disorder, but many students will express themselves more weakly in languages other than their mother tongue ....

Rob: I find this line of reasoning complete nonsense. Obviously, such a test cannot form the basis for a final tenth-level grade assessment. I agree on that. But any assessment until those final exams must be regarded as a process!

After consulting school management, the teachers decide to develop (among other things) a project on terrorism with the English teachers. They solve the assessment issue by compromising between different considerations. All students must conduct an oral presentation about terrorism in English. In addition, the students must conduct a 'subject conversation' to illustrate their ability to use core concepts, but this can be done either in English or in their mother tongue, depending on what the students themselves choose.

What, therefore, forms the grounds for this decision? Let me first turn to the knowledge resources at play. One resource is the school's strategy paper and its underlying premises and curriculum frame. In addition, a range of conceptual and material assessment resources are evoked. The reference to the EPC appears as a standard, and in this sense, as a knowledge resource, both in a legal and epistemic sense.

What space of action is enabled through the assemblage of these resources? First, the call for cooperation between subjects in the strategy document is closely linked with 'teaching with big ideas,' which is a conceptual resource that was referred to regularly during the interactions between the members of the social sciences team. This resource incorporates ideas of teaching to achieve 'deep understanding.' Rather than covering the many fragments of content, the aim is to uncover the big ideas in a given field that are framed by the teachers as linking together different activities 
(assessment, content, subjects, and methods) in ways that provide meaning for students. Second, resources related to AfL had been a priority focus in the past decade. Teachers were expected to design 'assessment packages' for the purposes of documenting learning, rather than for grading. These resources presuppose that the documentation of learning is conducted through a wide range of subject activities and that these are regarded as a process, as in Rob's final statement. In other words, among assessment resources, many are incorporated with an implicit understanding that assessment is to be adapted to different contexts and learning situations. Hence, the idea of merging assessments is interlinked with an understanding of assessments as integrated into the learning process and is aimed at achieving a more procedural approach to feedback.

With the reference to the ECPS, the idea of merging assessments is challenged. Students who perform poorly in language subjects should not have this issue spill over into poor assessment in a different subject, such as the social sciences. The students' self-determination is given weight, as in statements such as 'Unless the students themselves want to.' The reference to the ECPS acts as a standard that increases the complexity of the problem, as this is anchored in other considerations, materialities, and modes of knowledge — namely students' rights to participate in assessment work. ${ }^{2}$ At the moment when the legal side of assessment knowledge is emphasised, the separation between summative and formative assessment is challenged. Can the negative backlash from summative assessment in the assessment process be converted to positive support for formative assessment? With the presence of EPCS, the underlying claim is that within assessment as a process, there is an implicit summative assessment. ${ }^{3}$

At the intersection of the various framings and foregroundings of assessment, a space of contestation arises. In this space, competing epistemologies struggle for legitimacy. What is implied by regarding assessment as a process? Final exams rest on common standards and objective criteria that apply to the whole student cohort, while process assessments are about the learning path of and support for the individual student that are developed over time by the teacher and whose aims are to take many contexts and strategies into consideration. Moreover, the processual aspects of AfL incorporate the idea of students' participation in the assessment of their own learning processes, as

\footnotetext{
${ }^{2}$ These are anchored in The Regulations of Education Act on Assessment \$3. (This regulation is stronger in Norway than in most other countries [see, for instance, Hopfenbeck, Flóres, and Tolo 2015].)

${ }^{3}$ See, for instance, Tara (2007) for a discussion about the separation between formative and summative assessment.
} 
underscored here by the EPCS. But who should determine the 'right' method of assessment? Students? Teachers? External agents? Within the space of contestation, the teachers negotiate the frameworks for their professional responsibilities. Adding force to their justification, they respond with a form of agency by rejecting a 'ban' that is established by an external agent — the EPCS — as, according to Rob's statement, 'complete nonsense.'

Thus, assessment situations, which previously applied to individual teachers of single students, are raised to a principle level during the planning phase and involve important decisions. This space enables the teachers' consideration of the standards underlying the assessment practices, their implications, and whether they should be changed.

\section{Summary of findings}

The analysis of the data from the school level showed multiple knowledge resources circulating in and through the school that incorporated competing perspectives on how to frame the 'student,' 'assessment,' or 'learning objectives'; these were delegated to the teachers for them to make sense of and move towards curriculum solutions. The analysis of the data from the team level showed how the partial aspects identified in the two episodes required agentic strategies which took the form of exploring implications, negotiating among alternatives, and modifying the issue at hand (see Table 1). Both episodes showed how the teachers had to handle several sets of dilemmas that followed in the wake of tensions between materialised knowledge forms in order for them to make decisions and develop the subject curriculum further. By assembling resources that embody different rationalities, spaces of action emerged, thereby enabling them to find strategies for handling the issue at hand.

Table 1 depicts the epistemic conditions for teachers' decisions in local curriculum work. These processes were not enabled through the teachers' decisions alone but were also facilitated by all the conditions shown in Table 1. These included their framing of the task at hand, the resources they engaged with, and not least, the possible ways of thinking and acting that these knowledge resources triggered. The present study showed how all of these elements had to be negotiated by the teachers and that the teachers' strategies were important. 


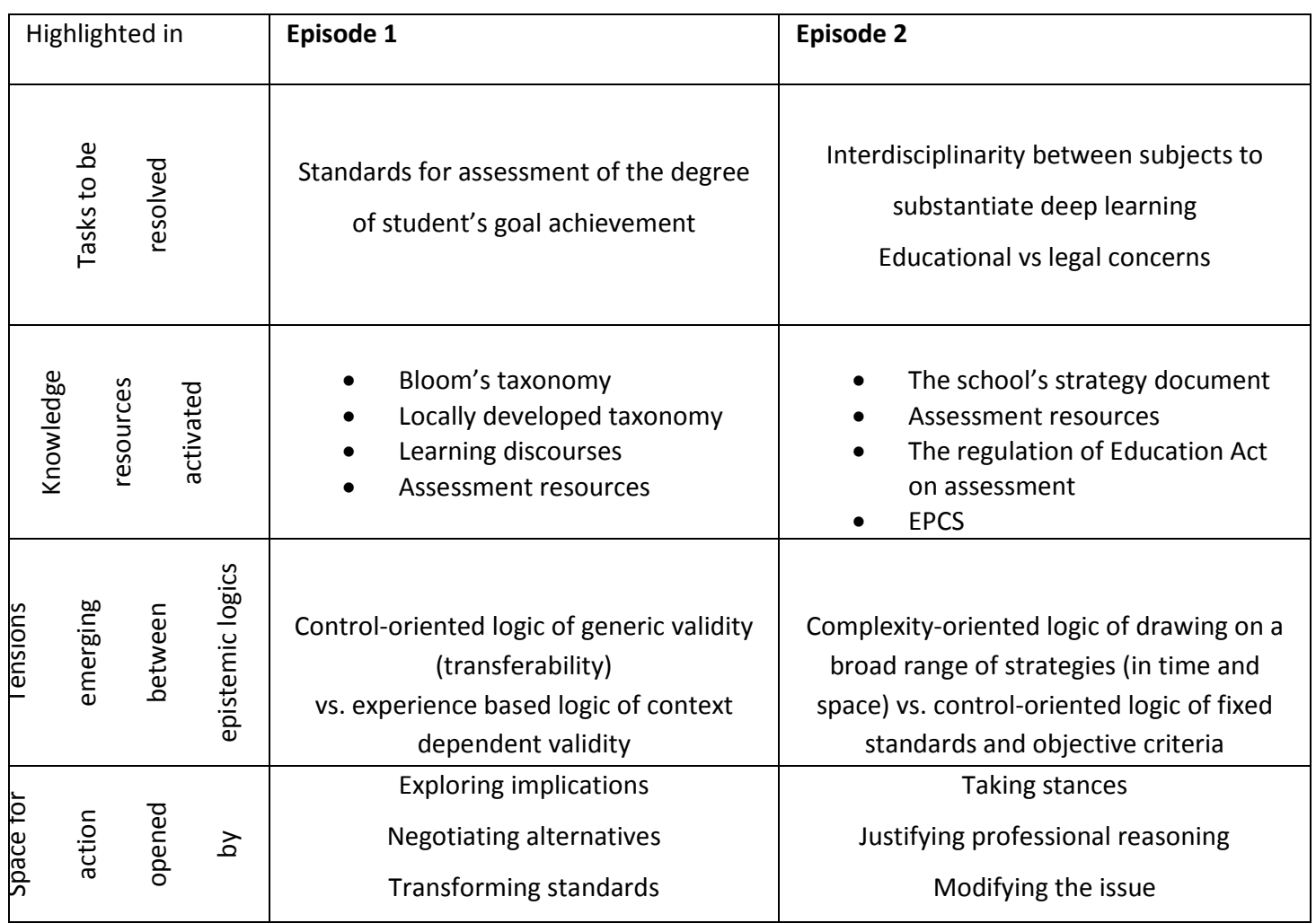

Table 1 Epistemic conditions for decision-making in local curriculum development

\section{Discussion: From dilemmas to decision-making}

In this study, I have explored teachers' work with local curriculum development by analytically tracing teachers' decision-making processes in dilemmatic situations. Rather than examining the kinds of decisions they made, I investigated what informed their actions and, eventually, what enabled their decisions. The analysis has shown that curriculum development is a multifaceted endeavour that involves the mobilisation and enactment of different knowledge resources in ways that require nuanced skills, responsibilities, and risks.

In this concluding discussion, I will relate the findings to aspects that seem particularly relevant to an understanding of teachers' decision-making processes within the context of curriculum work. First, while previous research has shown how conflicting concerns and contradictory expectations (Jonasson et al. 2015; Singh et al. 2015) may cause dilemmas in teachers' curriculum work; this analysis has offered a novel account of how epistemic dilemmas occur in teachers' curriculum construction by attending to the expanse of materialized knowledge representations available for teachers. The findings showed how the knowledge resources circulating in the school context influenced the teachers' decisions, but these decisions also involved 
modifications and the further development of resources which acted back on the school context. While research has typically been focused on either the school level or the teacher level, this study unravels the knowledge dynamics that connect levels by accounting for the constitutive role of knowledge resources. Nerland and Jensen (2014) discuss how knowledge resources act as linkages between the fields of work and knowledge production. In my findings, the spatial outreach of knowledge resources as AfL and Bloom's taxonomy expanded into the broader professional field.

Second, as Datnow and Hubbard (2016) also discussed, teachers' mastery of the vast number of new resources at their disposal requires an in-depth understanding of curriculum matters and students' learning processes. Similarly, Sing et al. (2015) found that a prerequisite for teachers to reassert their agency over the knowledge resources in use (in their case, student data) is the possibility of simultaneously engaging with and distancing themselves from the resources and their own teaching practices. Such double movements also appeared in my data when the teachers identified the underlying logics of knowledge resources in use, which indeed required in-depth understanding and exploration. However, by highlighting the relational and spatial dimensions of dilemmas (Fransson and Grannäs 2013), it was possible to not only recognise this as a matter of subject mastery but also, and equally importantly, to acknowledge what the knowledge resources mobilised. By illuminating the interaction between the teachers' mastery and the activation of resources, it was possible to see how this created spaces of action that formed the basis for the teachers' decision-making. To enable further understanding of the mechanisms through which this process evolved, I now turn to the contributions of the knowledge resources and the teachers, respectively.

First, as shown in the findings, knowledge resources come with specific logics and expectations regarding the correct methods of applying knowledge, or what I, using Kastenhofer's terminology, refer to as 'epistemic orientations.' These orientations are, however, 'ideal types' (Kastenhofer 2007) that do not exist in pure forms. They are expressed in various degrees and combinations that are performed and developed in different relations, as shown in this study. The identification of diverse epistemic orientations embodied in knowledge resources and the relationships of which they are part enables a detailed discussion of the specific roles of various knowledge forms and the tensions they may generate. It was precisely the variation between epistemic orientations and the limitations, possibilities, and complementarities they generated 
which gave rise to productive consolidations in the teachers' decision-making. The relationships between them assembled various epistemological points of view, or what Massey (2005) describes as 'spheres of possibility,' that constituted spaces of action through interactional moments.

Second, the complexity that comes with divergent epistemic orientations imposed changing demands on teachers regarding how to handle dilemmas. The analysis shows how teachers' capacity to explore and choose among seemingly incompatible alternatives required agency in the form of extended responsibilities taken on by the teachers. The epistemic orientations incorporated into the knowledge resources had effects but were simultaneously translated and modified by the teachers. Hence, knowledge resources coming from a control-oriented field did not necessarily end up as control oriented when applied, because the space for action enabled transformations.

The findings presented above also allow for some methodological reflections on how such processes can be explored. Investigating the complex interaction between knowledge forms and teachers' strategies required micro-level analysis at the action level. This enabled the documentation of teachers' decision-making as it unfolded and points to the ways in which dialogue was an important driver that was capacitated by negotiations of the various conditions and resources activated.

\section{Conclusions and implications}

As the inflow of knowledge resources to improve education increases, teachers' work and responsibilities become more complex and burdened with dilemmas. What implications might this have for schools and teachers? First, there is a need to recognise the types of epistemic engagement that has been revealed here as important to teachers' professional competencies. Schools and decision-makers at all levels must recognise the dilemmas that are delegated to teachers to handle, and teachers must be given the necessary time and space to work with them. As shown above, collective responsibility requires leadership and the extensive coordination of time and tasks across the school. The teachers I followed for this research were, for the most part, experienced and had high levels of professional expertise. However, the decisions they made and the dilemmas they handled could not have been accomplished by individual teachers alone but, rather, demanded collective knowledge work. Finally, although qualifying teachers 
for the complex tasks described above poses challenges, the empirical analysis presented here illustrate good reasons to include curriculum construction processes as important learning activities in teacher education programmes. Teachers' work is rooted in multiple - often contradictory - knowledge forms and sources that need to be aligned in the performance of curriculum development. More research is needed to unveil how these processes are enacted in work and educational settings.

\section{References}

Andriopoulos, C. and M. Gotsi. 2017. "Methods of paradox", in Lewis, M., Smith, W., Jarzabkowski, P. and Langley, A. (Eds), Handbook of Organizational Paradox: Approaches to Plurality, Tensions and Contradictions, 513-528. Oxford UK: Oxford University Press.

Apple, M. W. 2007. "Ideological Success, Educational Failure? On the Politics of No Child Left Behind." Journal of Teacher Education 58 (2): 108-116.

Asdal, K. 2015. "What Is the Issue? The Transformative Capacity of Documents." Distinktion: Scandinavian Journal of Social Theory 16 (1): 74-90.

Ball, S. J. 2005. Education Policy and Social Class: The Selected Works of Stephen J. Ball. Abingdon: Routledge.

Ben-Peretz, M., and M. A. Flores. 2018. "Tensions and Paradoxes in Teaching: Implications for Teacher Education." European Journal of Teacher Education 41 (2): 202-213.

Boschman, F., S. McKenney, and J. Voogt. 2014. "Understanding Decision Making in Teachers' Curriculum Design Approaches.” Educational Technology Research and Development 62 (4): 393-416.

Ceulemans, C., M. Simons, and E. Struyf. 2012. "Professional Standards for Teachers: How Do They 'Work'? An Experiment in Tracing Standardisation in-theMaking in Teacher Education.” Pedagogy, Culture \& Society 20 (1): 29-47.

Cho, V., and J. C. Wayman. 2014. 'Districts' Efforts for Data Use and Computer Data Systems: The Role of Sensemaking in System Use and Implementation." Teachers College Record 116 (2): 1-45.

Czarniawska-Joerges, B. 2007. Shadowing: And Other Techniques for Doing Fieldwork in Modern Societies. Copenhagen: Copenhagen Business School Press DK. 
Czerniawski, G. 2011. "Emerging teachers-emerging identities: Trust and accountability in the construction of newly qualified teachers in Norway, Germany, and England." European Journal of Teacher Education 34 (4): 431447.

Datnow, A., and L. Hubbard. 2016. "Teacher Capacity for and Beliefs about DataDriven Decision Making: A Literature Review of International Research." Journal of Educational Change 17 (1): 7-28.

Derry, S. J., R. D. Pea, B. Barron, R. A. Engle, F. Erickson, R. Goldman, and B. L. Sherin. 2010. "Conducting Video Research in the Learning Sciences: Guidance on Selection, Analysis, Technology, and Ethics.” The Journal of the Learning Sciences 19 (1): 3-53.

Fenwick, T., R. Edwards and P. Sawchuk. 2011. Emerging Approaches to Educational Research: Tracing the Socio-Material. Abingdon: Routledge.

Fenwick, T., E. Mangez, and J. Ozga, eds.2014. World Yearbook of Education 2014: Governing knowledge: Comparison, knowledge-based technologies and expertise in the regulation of education. 4-9. London: Routledge.

Fransson, G., and J. Grannäs. 2013. "Dilemmatic Spaces in Educational Contexts: Towards a Conceptual Framework for Dilemmas in Teachers Work.” Teachers and Teaching 19 (1): 4-17.

Gherardi, S., and A. Strati. 2012. Learning and Knowing in Practice-Based Studies. Cheltenham: Edward Elgar.

Hardy, I., and S. Lewis. 2018. "Visibility, Invisibility, and Visualization: The Danger of School Performance Data.” Pedagogy, Culture \& Society 26 (2): 233-248.

Hargreaves, A. 2003. Teaching in the Knowledge Society: Education in the Age of Insecurity. New York:Teachers College Press.

Hermansen, H., and M. Nerland. 2014. "Reworking Practice through an AfL Project: An Analysis of Teachers' Collaborative Engagement with New Assessment Guidelines." British Educational Research Journal 40 (1): 187-206.

Hirsh, Å. 2014. "The Individual Development Plan: Supportive Tool or Mission Impossible? Swedish Teachers' Experiences of Dilemmas in IDP Practice.” Education Inquiry 5(3): 405-427.

Hopfenbeck, T. N., M. T. Flórez Petour, and A. Tolo. 2015. "Balancing Tensions in Educational Policy Reforms: Large-Scale Implementation of Assessment for 
Learning in Norway.” Assessment in Education: Principles, Policy \& Practice 22(1): 44-60.

Jonasson, C., A. Mäkitalo, and K. Nielsen. 2015. “Teachers' Dilemmatic DecisionMaking: Reconciling Coexisting Policies of Increased Student Retention and Performance.” Teachers and Teaching 21 (7): 831-842.

Kastenhofer, K. 2007. “Converging Epistemic Cultures? A Discussion Drawing on Empirical Findings.” Innovation 20 (4): 359-373.

Markauskaite L., and P. Goodyear. 2017. "Inscriptions Shaping Mind, Meaning and Action." In Epistemic Fluency and Professional Education: Professional and Practice-Based Learning. Vol. 14: 303-339. Dordrecht: Springer.

März, V., G. Kelchtermans, and K. Vermeir. 2017. “Artifacts as Authoritative Actors in Educational Reform.” Journal of Educational Change 18 (4): 439-464.

Massey, D. 2005. For Space. London: Sage.

Nerland, M. and K. Jensen. 2014. "Changing Cultures of Knowledge and Professional Learning." In S. Billet, C. Harteis and Gruber (Eds.), International Handbook of Research in Professional and Practice-Based Learning, 611-640. Dordrecht: Springer.

Nespor, J., 2003. “Undergraduate Curricula as Networks and Trajectories.” In Space, Curriculum and Learning, edited by R. Edwards and R. Usher, 93-108 Greenwich, CT: Information Age Publishing.

Patton, M. Q. 2002. Qualitative Research and Evaluation Methods. 3rd ed. Thousand Oaks, CA: Sage.

Priestley, M, S. Minty, and M. Eager. 2014. "School-based curriculum development in Scotland: Curriculum policy and enactment." Pedagogy, Culture \& Society 22 (2): 189-211.

Read, S., and J. Swarts. 2015. "Visualizing and Tracing: Research Methodologies for the Study of Networked, Sociotechnical Activity, Otherwise Known as Knowledge Work." Technical Communication Quarterly 24 (1): 14-44.

Remesal, A. 2011. "Primary and Secondary Teachers' Conceptions of Assessment: A Qualitative Study." Teaching and Teacher Education, 27 (2): 472-482.

Ryan, G. W., and H. R. Bernard. 2003. “Techniques to Identify Themes.” Field Methods 15 (1): 85-109. 
Scott, W. R. 2014. Institutions and Organizations: Ideas, Interests, and Identities. 4th ed. Thousand Oaks, CA: Sage.

Singh, P., M. Märtsin, and K. Glasswell. 2015. "Dilemmatic Spaces: High-Stakes Testing and the Possibilities of Collaborative Knowledge Work to Generate Learning Innovations.” Teachers and Teaching 21(4): 379-399.

Tara, M. 2007 “Machinations of Assessment: Metaphors, Myths and Realities.” Pedagogy, Culture \& Society 15 (1): 55-69.

Vanden, BL., and M. Simons. 2017. "School Stuff: A Pedagogical Regime of Enunciation?" Pedagogy, Culture \& Society 25 (1): 105-119.

Vermeir, K., G. Kelchtermans, and V. März. 2017. "Implementing Artifacts: An Interactive Frame Analysis of Innovative Educational Practices.” Teaching and Teacher Education 63: 116-125.

Voogt, J., M. Jules M. Pieters, and Adam Handelzalts. 2016. "Teacher collaboration in curriculum design teams: effects, mechanisms, and conditions." Educational Research and Evaluation 22 (3-4): 121-140.

Wall, L. 2017. "Institutional Logics and Curriculum Decision Making: Enacting the Australian Curriculum English and NAPLAN Literacy." The Australian Educational Researcher 44 (4-5): 391-407.

Yanow, D., S.Ybema, and M.van Hulst. 2012. "Practicing organizational ethnography", in G. Symon and C. Cassell (Eds.), Qualitative Organizational Research. 331335. London: Sage. 